\title{
Optimal Placement of DG for Power Loss Minimization: A Review
}

\author{
Gunjan Sharma, Sarfaraz Nawaz \\ Department of Electrical Engineering, Swami Keshvanand Institute of Technology, Management \& Gramothan, Jaipur- \\ 302017 (INDIA) \\ Email: sarfaraz@skit.ac.in \\ Received 25.07.2021, received in revised form 29.10.2021, accepted 29.10.2021 \\ doi: 10.47904/IJSKIT.11.2.2021.55-62
}

\begin{abstract}
Distributed generation is the future of electrical energy because the fuels (such as coal) used in conventional methods are depleting day by day. The demand for electricity is increasing rapidly. Hence, consumption of conventional fuels is also increasing resulting in depletion of these resources and high carbon emissions. Also, the harmful impacts caused by these resources has shifted the focus towards more ecofriendly methods i.e. renewable sources of energy. Integrating these renewables with existing system is the new trend. Various studies have been carried out discussing the merits and demerits of DG integration with the central grid. The process of adding distributed generators with the power system is quite challenging. The DG's should be added such that it enhances the system performance and increases reliability. This paper discusses about the optimal placement of DG to reduce power losses in the network.
\end{abstract}

Keywords- Distributed generation, Power losses, DG penetration, Optimal placement, Voltage stability.

\section{INTRODUCTION}

Distributed generation also known as decentralized generation or embedded generation is broadly defined as the generation of electricity at or near the site of consumption. The method of generation usually involves renewable resources; therefore, these are referred as distributed energy resources. Renewable energy sources are the current trends in electricity generation (mainly hydropower) because they are present in abundance and also cause no harm to the environment. The hydropower alone contributes to the $62.83 \%$ of total world renewable energy generation followed by wind (19.03\%), solar $(8.76 \%)$ and others. The countries across the globe are targeting to synchronize the DG's with the existing grids to reduce the carbon emission and other harmful impacts of conventional generation. Integrating such type of generation units with existing grid helps not only in reducing losses but also enhances the power quality by improving the voltage profile. But the major problem arises when the integration level increases and reaches a value where these DG starts showing a negative impact on the system. The penetration of DG into the grids is a major concern because when more load is shifted to DG's, the load on existing generating units is reduced which leads to various unbalancing problems in the system. Hence, before planning any system it is important to study whether it can cope with increasing use of DG or not. The existing grids were not designed to accommodate DG, but with time, the use of DG has increased and hence, the system should be updated accordingly.

There are mainly four types of DG's

1. Type 1: DG which inject both active power and reactive power in the system.

2. Type 2: DG capable of injecting only active power.

3. Type 3: DG delivering reactive power only.

4. Type 4: DG which absorb reactive power and inject active power in the system.[1]

The methods of generation can vary from solar photovoltaics, wind energy conversion system to fuel cells, diesel engines etc. Each method has a different impact on the power system. The negative impact includes the voltage quality degradation, induction of harmonics in the system, increased voltage fluctuations on the consumer side which further results in poor power quality, voltage stability issues, power system inertia, increasing fault currents, false current detection by power system protection equipment.

Penetration level of DG in the system is obtained by,

$$
\% D G \mathrm{P}=\frac{P_{\mathrm{DG}}}{P_{\mathrm{DG}}+P_{\mathrm{CG}}} \times 100
$$

Where, $\% D G \mathrm{P}=$ percentage of $\mathrm{DG}$ penetration

$P$ DG $=$ power generated by DG

$P$ CG $=$ power generated by centralized grid

Studies show that siting of DG in the system is a crucial factor to determine its impact on the grid. Inappropriate placement of DG in the network leads to overvoltage and phase imbalances. However, when placed at suitable nodes, it enhances the voltage profile and phase imbalance is comparatively low. [2] Integration of DG with a weak distribution network causes a voltage rise during low load demand. Although, by strengthening the overhead lines and placing a shunt reactance at critical points, the electrical power losses are reduced along with the enhancement of voltage profile. [3] Large integration of DG also results in injection of high short circuit currents in the system. At an appropriate penetration level, the short circuit currents are not of much concern in reference to protection system. [4]. Due to uncertainty in load demands, power system utilities are facing problems related to control and stability of the system. Hence, it is important to improve the power quality of the system by integrating it with 
DG. But integration of DG in the system such that the system remains balance is a challenge for utilities and researchers as well. To enhance the system performance, the location of distributed generators along with proper size should be used. Many researchers observed various ideal location in different test system considering multiple factors to add DG in the system. Neenu Rose Antony et. Al [5] obtained the ideal location of DG by enhancing voltage stability. Satish Kumar Injeti et al. placed DG along with plug in electric vehicle to enhance voltage profile and minimize power losses. [6] Mir Emad Hamidi et al. reduced the negative impact of DG on distribution system by placing it with fault current limiter. [7] M.M. Aman used approach based on power stability index. [8]Galiveeti Hema kumar Reddy et al placed DG to enhance system reliability and response. [9] Various studies have been carried out to improve voltage stability along with decrease in power losses. [10][11][12][13][14][15] This paper focuses on the researches carried out so far on optimal placement of DG in order to minimize power losses in the system.

\section{IMPORTANCE OF DG SIZING AND SITING}

Various studies have been carried out discussing the impacts of placing a DG at different nodes in the system. These studies suggested that placement of DG is an important factor to extract the maximum benefit from the system. The size of DG along with suitable location is another crucial factor for enhancing power system performance. The best location depends on the type of DG used. When integrating a single-phaseDG, the allocation should be done at varying phases such that the phase imbalance limits are not violated. The size of DG defines the penetration limit and varies with system. [2]

\section{FACTORS CONSIDERED FOR OPTIMAL PLACEMENT OF DG}

\subsection{DG Penetration Factor (DGPF):}

Losses are required to be minimized in order to achieve a healthy and stable operation power system. These losses are either active power losses or reactive power losses. The power losses between line (i) and (i+1) are given by,

$$
\mathrm{P}_{\text {loss }}(i, i+1)=\frac{\left(P_{i}^{2}+Q_{i}^{2}\right)}{\left|V_{i}^{2}\right|} \times r(i, i+1)
$$

where, $\mathrm{P}_{\mathrm{i}}$ is active power flow at bus $\mathrm{i}$ and $\mathrm{Q}_{\mathrm{i}}$ is reactive power flow at bus $\mathrm{i}[5]$. High penetration of DG results in more reduction of losses, but penetration of DG beyond a certain limit can adversely affect the power system.

\subsection{Voltage Profile}

What impact will DG have on system voltage profile depends on how much active and reactive capacity the DG has. Overvoltage caused due to DG can be avoided by making slight adjustments in capacitor switching on the node at which DG is placed.[2] The voltage can be controlled either by controlling devices or by properly designing the system.

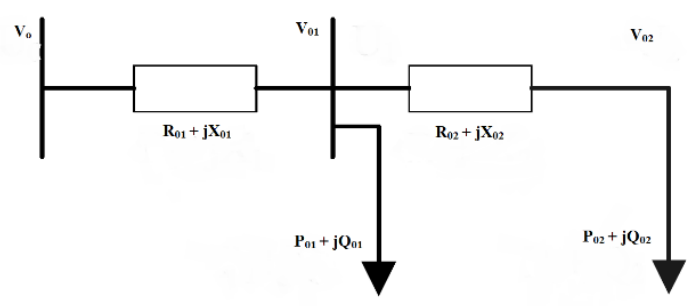

Fig.1 Sample network

The voltage drop between the lines can be given as,

$$
\Delta V_{i}=\frac{P_{0 i} R_{0 i}+Q_{0 i} X_{0 i}}{V_{r}}
$$

$\mathrm{V}_{\mathrm{r}}$ is the rated system voltage. The voltage at node 2 is given as,

$$
\begin{aligned}
V_{02}=V_{0}-\Delta V= & V_{0}-\frac{P_{01} R_{01}+Q_{01} X_{01}}{V_{r}} \\
& +\frac{P_{02} R_{02}+Q_{02} X_{02}}{V_{r}}
\end{aligned}
$$

With addition of DG at bus 1 , the voltage at node 2 becomes,

$$
\begin{aligned}
& V_{02}=V_{0}-\Delta V \\
& =V_{0}-\frac{\left(P_{01}-P_{d g}\right) R_{01}+\left(Q_{01}-Q_{d g}\right) X_{01}}{V_{r}} \\
& +\frac{P_{02} R_{02}+Q_{02} X_{02}}{V_{r}}
\end{aligned}
$$

From above equation, it can be derived that the voltage levels in the system varies with variation in integration level and position of the distributed generation in the power system. The penetration level of DG in the system should be such that it enhances the system voltage profile. [16]

\subsection{Transient stability of system}

When a system is subjected to a large disturbance, it tends to return to its initial stable conditions. This ability of power system is known as transient stability of system. The penetration of DG means meeting the increased load demand with DG or by supplying the increasing demand caused by unit outage due to any reason. The transient can be analysed by analysing the maximum deviation in rotor speed and the duration of oscillations during and after fault. The weak connection between generators and loads on a heavily loaded line causes large oscillations. Hence, increased power flow has a harmful effect on the 
damping of oscillations. Integrating DG with controllers can reduce oscillations in the system.[35] The equations showing the unbalancing between mechanical and electromagnetic torque of a single machine for transient stability analysis can be given as,

$$
\frac{d \omega}{d t}=\frac{1}{2 H}\left(T_{m}-T_{e}-K_{d} \omega\right)
$$

Where $T_{m}$ and $T_{e}$ are mechanical and electromagnetic torque respectively. $\mathrm{K}_{\mathrm{d}}$ is the damping factor, $\mathrm{H}$ denotes inertia constant and $\omega$ represents angular velocity of rotor. This equation is applicable to DG using synchronous generators, wind turbines etc. Since, there is no moving part in solar generation therefore there is no inertia. The above equation in terms of power is given as,

$$
\frac{d \omega}{d t}=\frac{1}{2 H}\left(P_{m}-P_{e}-K_{d} \omega\right)
$$

Where $\mathrm{P}_{\mathrm{m}}$ and $\mathrm{P}_{\mathrm{e}}$ is the input mechanical power and output electrical power respectively. [17]

\subsection{Fault current}

During a fault, the DG contributes to the fault current, which leads to malfunctioning of the system and affecting the system security and reliability. Studies show that a fault occurring at generation node contributes to maximum fault current ratio (ratio of fault current with DG and without DG) whereas at low voltage nodes the impact of fault current is significantly low. [2]

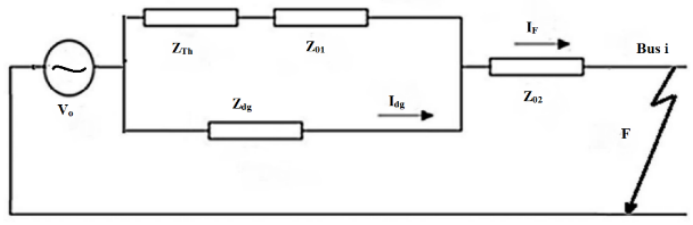

Fig.2.Sample model for short circuit

In above figure, $\mathrm{I}_{\mathrm{F}}$ represents fault current at bus $i$, which is given as,

$$
I_{F}=\frac{V_{\mathrm{o}}}{\frac{\left(Z_{T h}+Z_{01}\right) \times Z_{d g}}{Z_{T h}+Z_{01}+Z_{d g}}+Z_{02}}
$$

Where $Z_{01}$ and $Z_{02}$ are line impedances, $Z_{T h}$ is Thevenin equivalent. $Z_{\mathrm{dg}}$ is the impedance of DG. [7]

\subsection{Economical factors}

These factors are considered in order to reduce the DG investment, operation and maintenance cost. For example, the ideal location for setting up solar plant is where sufficient amount of sunlight is available throughout the year in order to maintain smooth operation of the plant. Similarly, wind plants must be placed where winds at desirable speed flows. Srinivasa Rao Gampa et. al. used cost ratio to calculate the most economical size of DG which is given as,

$$
C R=\frac{\sum C_{d g} P_{d g}}{\sum C_{d g} \times P_{d g}^{\max }}
$$

$\mathrm{C}_{\mathrm{dg}}$ represents the cost of $\mathrm{DG}$ and $\mathrm{P}_{\mathrm{dg}}$ represents the power generated from DG. $P_{d g}^{\max }$ denotes the maximum power generated from DG.[18] Considering economy, the investment includes the cost to set up the DG and the benefit obtained from it. [19] Benefits include reduction in emissions, environmental effects etc.

\section{OPTIMIZATION TECHNIQUES USED IN DG ALLOCATION}

\subsection{Metaheuristic approaches}

Based on diversifying and exploring these algorithms have been used in past to solve different optimization issues. Krischonme Bhumkittipich et al. [36] used Particle Swarm optimization technique to reduce power losses by integrating and optimally placing DG in the system. PSO was given by Kennedy and Eberhart in 1995 and since then it has been widely used to solve various optimization problems. [37][23][24]. An algorithm based on genetics is another technique to provide solutions to problems. Researchers have used these algorithms to reduce the time of computation. [30][36][28]. Other approaches consist of Ant colony optimization, Artificial Bee colony, Tabu search, Bat algorithm, Modified Shark Smell optimization etc. [38][39][40][41][42][43]

\subsection{Heuristic approaches}

Such approaches are employed where approximate solutions are acceptable. Sara YuliethBocanegra et al. [44] used this approach to obtain the ideal location and size of distributed generation in network system. Similarly, Sheeraz Kirmani et al [45] used this approach to correctly place the distributed generators in network system in order to reduce the power system losses[20]

\subsection{Analytical approaches}

Since, these approaches do not use iterations, therefore problems associated with it such as convergence are also absent. [46] ParthaKayalet al used a method based on voltage stability and loss sensitivity factor to derive the best location and size for distributed generation in the system. Tuba Gözel et al. optimally placed DG using loss sensitivity factor approach. [47][48][20][22]

\subsection{Hybrid approaches}

Hybrid approach is when two or more optimization techniques are combined together to obtain more accurate result with a smaller number of iterations.[20] Ambika Ramamoorthy et al. used combined particle swarm and GSA for enhancing the voltage profile and minimizing power losses. [23] Another hybrid approach for identifying best location 
to place DG is combined Clonal algorithm and PSO. [24] Similarly, various other examples of hybrid approaches are Grasshoper and cuckoo search optimization methods, PSO and Improved analytical method, PSO and honey bee mating optimization, Grey wolf optimization and PSO, etc. [11][25] [26] [27][28]

Table 1: Comparative study of loss minimization for 33-bus

\begin{tabular}{|c|c|c|c|c|c|c|}
\hline & & & syst & & & \\
\hline 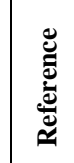 & 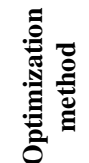 & 总 & 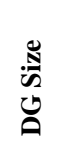 & 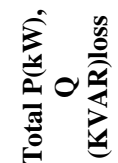 & 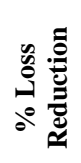 & 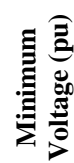 \\
\hline
\end{tabular}

\begin{tabular}{|c|c|c|c|c|c|c|}
\hline \multicolumn{7}{|c|}{ With one DG } \\
\hline$\sqrt{\tilde{\Omega}}$ & 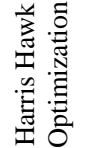 & - & $\begin{array}{l}\stackrel{m}{+} \\
\overrightarrow{0} \\
\text { ה. }\end{array}$ & 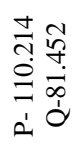 & $\begin{array}{l}\stackrel{R}{\sim} \\
\stackrel{\sigma}{2}\end{array}$ & $\begin{array}{l}\text { ఫे } \\
\text { aे }\end{array}$ \\
\hline & & & & & & \\
\hline
\end{tabular}

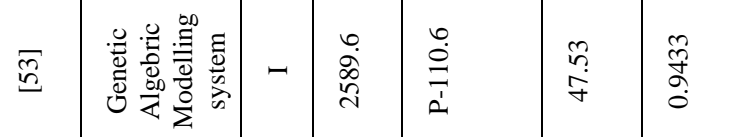

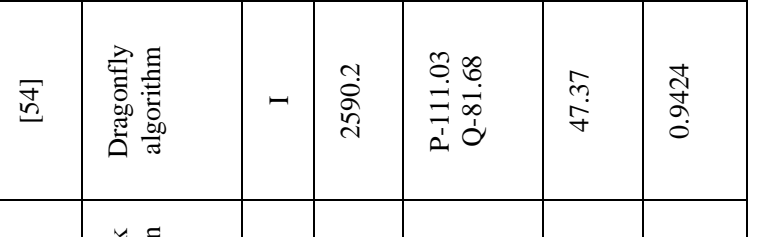

\begin{tabular}{|c|c|c|c|c|c|c|}
\hline & 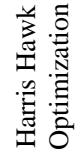 & $=$ & 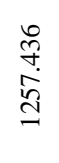 & 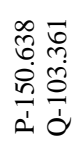 & $\underset{\substack{\sim \\
\sim}}{0}$ & $\begin{array}{l}\infty \\
\frac{0}{\sigma} \\
0\end{array}$ \\
\hline & 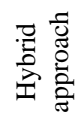 & $=$ & 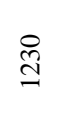 & $\begin{array}{l}\underset{7}{\vec{n}} \\
\stackrel{\vec{n}}{i}\end{array}$ & $\begin{array}{l}\text { d̦ } \\
\text { d. }\end{array}$ & ' \\
\hline
\end{tabular}

\begin{tabular}{|c|c|c|c|c|c|c|}
\hline 륨 & 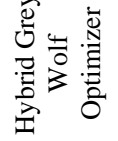 & $=$ & $\stackrel{\infty}{\stackrel{\infty}{\beth}}$ & 离 & 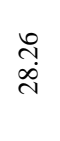 & $\begin{array}{l}\frac{n}{a} \\
0\end{array}$ \\
\hline
\end{tabular}

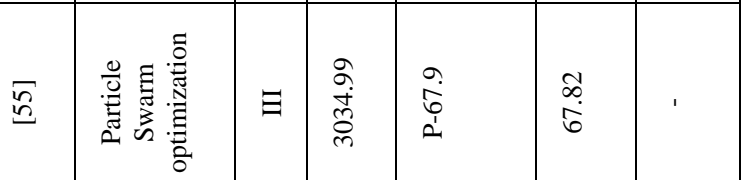

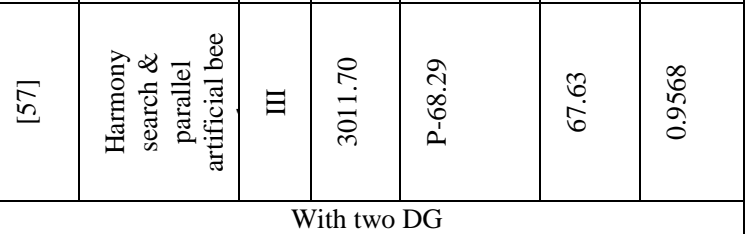

\begin{tabular}{|c|c|c|c|c|c|c|}
\hline $\begin{array}{l}\bar{\infty} \\
\stackrel{s}{\Omega}\end{array}$ & 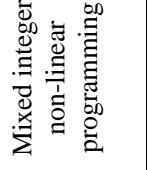 & - & ષ્ণి & 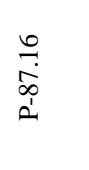 & $\begin{array}{l}\vec{a} \\
\text { id } \\
i n\end{array}$ & ' \\
\hline$\overline{\underline{6}}$ & 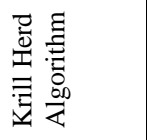 & - & $\begin{array}{l}\text { त̂̀ } \\
\text { ठํ. } \\
\text { ते }\end{array}$ & 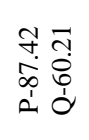 & 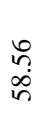 & $\begin{array}{l}\text { o. } \\
\text { ̊. }\end{array}$ \\
\hline $\bar{\Omega}$ & 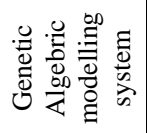 & - & 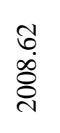 & $\begin{array}{l}\hat{0} \\
0 \\
\infty \\
0 \\
0 \\
.\end{array}$ & $\begin{array}{l}\tilde{\infty} \\
\infty \\
\infty \\
\infty\end{array}$ & $\begin{array}{l}\text { 趌 } \\
\text { o. }\end{array}$ \\
\hline $\begin{array}{l}\bar{\sigma} \\
\stackrel{n}{n}\end{array}$ & 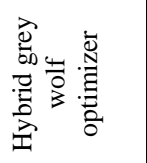 & $=$ & $\overrightarrow{\sqrt{n}}$ & 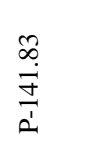 & 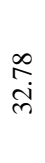 & $\begin{array}{l}\infty \\
\tilde{\delta} \\
\hat{\sigma} \\
\delta\end{array}$ \\
\hline 气્ & 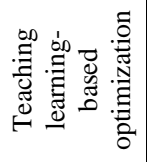 & $=$ & $\begin{array}{l}\hat{\imath} \\
\infty \\
\hat{\sim} \\
\end{array}$ & 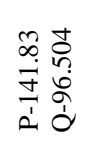 & 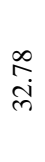 & $\begin{array}{c}\text { ô } \\
\text { ळ. } \\
\delta\end{array}$ \\
\hline 宫 & 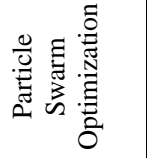 & $\exists$ & \begin{tabular}{l}
2 \\
o \\
\multirow{2}{*}{}
\end{tabular} & 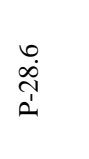 & 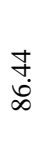 & ' \\
\hline స్ & 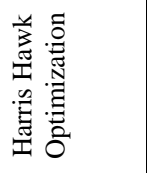 & $\exists$ & 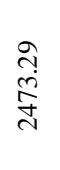 & 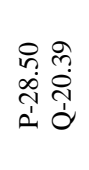 & 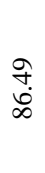 & $\begin{array}{l}\text { oे } \\
\text { o. } \\
o\end{array}$ \\
\hline \multicolumn{7}{|c|}{ With three DG } \\
\hline$\overline{8}$ & 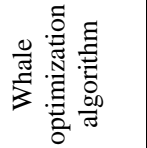 & $\tau$ & 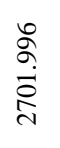 & 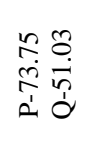 & $\begin{array}{l}\text { o. } \\
\dot{d i b}\end{array}$ & $\begin{array}{l}\infty \\
o \\
o \\
o \\
0\end{array}$ \\
\hline बू & 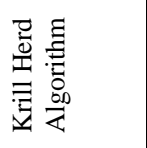 & - & 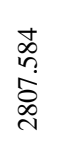 & 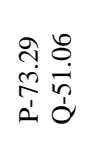 & 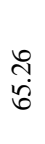 & 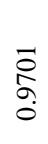 \\
\hline 点 & 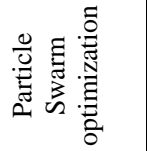 & - & ণ্ণ & $\begin{array}{l}\stackrel{\hat{~}}{\mathrm{i}} \\
\stackrel{\Lambda}{1}\end{array}$ & $\begin{array}{l}n \\
n \\
0\end{array}$ & ' \\
\hline $\bar{\sigma}$ & 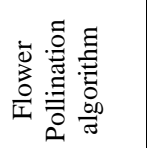 & $=$ & $\begin{array}{l}\text { ถે } \\
\text { הू }\end{array}$ & $\begin{array}{l}n \\
\delta \\
\dot{0} \\
\frac{1}{2}\end{array}$ & $\begin{array}{l}\hat{0} \\
\text { तें }\end{array}$ & $\begin{array}{l}\text { \&े } \\
\text { वे } \\
\text { o }\end{array}$ \\
\hline
\end{tabular}




\begin{tabular}{|c|c|c|c|c|c|c|}
\hline$\sqrt{\tilde{2}}$ & 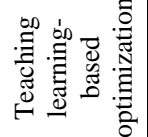 & $=$ & 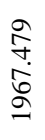 & 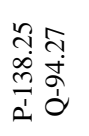 & $\begin{array}{l}\text { f } \\
\dot{m}\end{array}$ & $\frac{\infty}{\vec{\sigma}}$ \\
\hline $\begin{array}{l}n \\
\tilde{n}\end{array}$ & 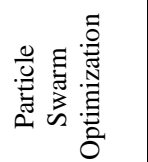 & $\Xi$ & $\begin{array}{l}\hat{\sigma} \\
\vdots \\
\text { i } \\
\text { o } \\
\text { d. }\end{array}$ & 京 & $\begin{array}{l}\underset{f}{+} \\
\dot{\alpha}\end{array}$ & \\
\hline ह & 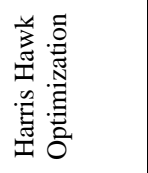 & $\exists$ & 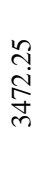 & 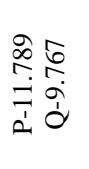 & $\begin{array}{l}\vec{f} \\
\dot{J}\end{array}$ & $\begin{array}{l}\text { శ్ } \\
\delta \\
\delta\end{array}$ \\
\hline
\end{tabular}

Table 2: Comparative study of loss minimization for 69-bus

\begin{tabular}{|c|c|c|c|c|c|c|}
\hline \multicolumn{7}{|c|}{ system } \\
\hline 苞 & 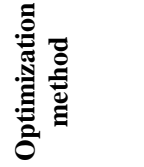 & $\frac{\mathscr{0}}{2}$ & 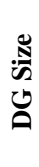 & 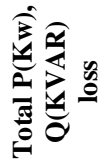 & 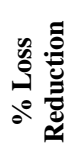 & 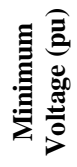 \\
\hline \multicolumn{7}{|c|}{ With one DG } \\
\hline$\widetilde{\widetilde{V}}$ & 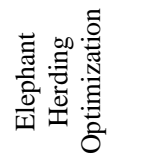 & ー & $\begin{array}{l}0 \\
\stackrel{\infty}{\infty}\end{array}$ & 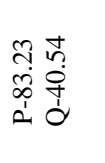 & $\hat{\sigma}$ & $\begin{array}{l}m \\
\infty \\
0 \\
0\end{array}$ \\
\hline$\underset{\nabla}{\mathscr{D}}$ & 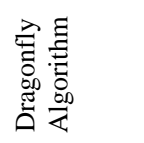 & レ & $\underset{\infty}{\stackrel{\infty}{\infty}}$ & 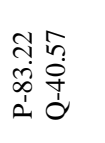 & $\begin{array}{l}\bar{\sigma} \\
\text { ஸુ }\end{array}$ & 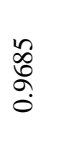 \\
\hline$\sqrt{n}$ & 导 & • & 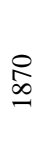 & $\begin{array}{l}\text { Ñ } \\
\text { ஸ் } \\
\infty \\
1\end{array}$ & $\begin{array}{l}\overline{0} \\
\text { लె }\end{array}$ & 1 \\
\hline 그 & 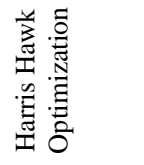 & $\models$ & 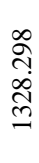 & 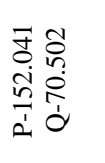 & 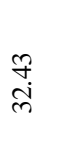 & $\begin{array}{l}\hat{o} \\
\text { on } \\
\hat{0}\end{array}$ \\
\hline సี & 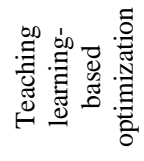 & $=$ & $\begin{array}{l}\mathfrak{a} \\
\text { ลे }\end{array}$ & 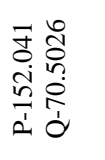 & $\underset{\stackrel{\Re}{\sim}}{\stackrel{m}{\sim}}$ & ồ \\
\hline $\begin{array}{l}\square \\
\curvearrowleft\end{array}$ & 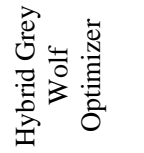 & $=$ & $\begin{array}{l}\text { mે } \\
\text { }\end{array}$ & $\begin{array}{l}\vec{J} \\
\text { i } \\
\frac{n}{2} \\
a^{\prime}\end{array}$ & 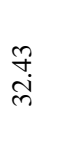 & $\frac{\bar{m}}{\grave{o}}$ \\
\hline
\end{tabular}

\begin{tabular}{|c|c|c|c|c|c|c|}
\hline$\overline{\widehat{\sigma}}$ & 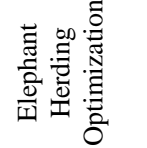 & B & $\begin{array}{l}\text { 今े } \\
\text { ते } \\
\text { ה̀ }\end{array}$ & 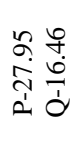 & 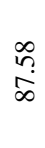 & $\begin{array}{l}\text { 芯 } \\
\text { Oे }\end{array}$ \\
\hline$\underset{n}{F}$ & 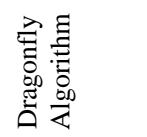 & 目 & 离 & 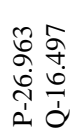 & 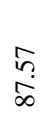 & 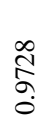 \\
\hline 气n & 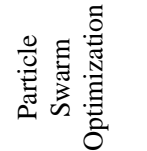 & 目 & $\begin{array}{l}\hat{\sigma} \\
\text { बे } \\
\text { ते }\end{array}$ & 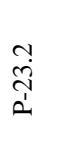 & $\begin{array}{l}\stackrel{a}{\circ} \\
\stackrel{\infty}{0}\end{array}$ & , \\
\hline \multicolumn{7}{|c|}{ With two DG } \\
\hline 호 & 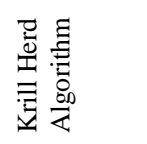 & - & $\begin{array}{l}\frac{\text { I }}{\mathrm{O}} \\
\text { Oे }\end{array}$ & 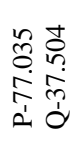 & $\begin{array}{l}\stackrel{0}{8} \\
\qquad b\end{array}$ & $\hat{a}$ \\
\hline$\tilde{n}$ & 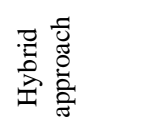 & - & 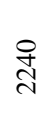 & 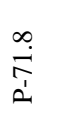 & $\begin{array}{l}\stackrel{a}{\infty} \\
\dot{\infty}\end{array}$ & ' \\
\hline$\sqrt[\widetilde{n}]{\underline{n}}$ & 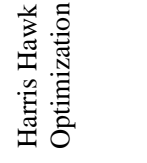 & - & $\begin{array}{l}\text { Oे. } \\
\text { त्र }\end{array}$ & 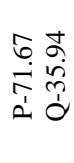 & 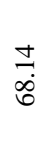 & $\begin{array}{l}\text { oे } \\
\text { ô } \\
\text { o. }\end{array}$ \\
\hline $\begin{array}{l}\mathscr{\bullet} \\
ٌ\end{array}$ & 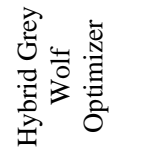 & $=$ & $\underset{\sigma}{\sigma}$ & 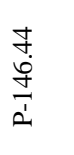 & $\begin{array}{l}\tilde{\alpha} \\
\dot{m}\end{array}$ & $\frac{n}{\sigma}$ \\
\hline$\sqrt[\widetilde{\sim}]{\approx}$ & 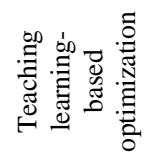 & $=$ & 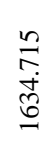 & 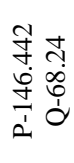 & $\begin{array}{l}\bar{a} \\
\dot{m}\end{array}$ & $\overrightarrow{\bar{\sigma}}$ \\
\hline$\stackrel{n}{n}$ & 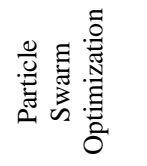 & 目 & 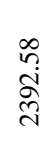 & 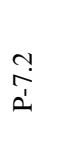 & $\begin{array}{l}\infty \\
\stackrel{\infty}{\infty}\end{array}$ & ' \\
\hline$\overline{\widetilde{N}}$ & 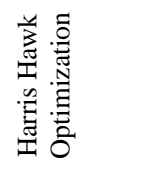 & $\Xi$ & $\begin{array}{l}\text { ते } \\
\text { fे } \\
\text { ָे }\end{array}$ & 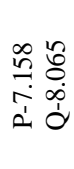 & \begin{tabular}{l}
0 \\
$\infty$ \\
$ه$ \\
\hdashline
\end{tabular} & $\begin{array}{l}\text { fे } \\
\text { gे } \\
O\end{array}$ \\
\hline \multicolumn{7}{|c|}{ With three DG } \\
\hline$\overline{\check{n}}$ & 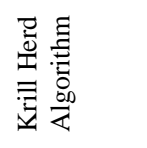 & - & $\frac{n}{m}$ & 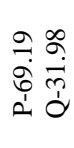 & $\begin{array}{l}\text { તે } \\
\text { bे }\end{array}$ & $\begin{array}{l}\tilde{\delta} \\
\hat{\delta} \\
\delta\end{array}$ \\
\hline
\end{tabular}




\begin{tabular}{|c|c|c|c|c|c|c|}
\hline $\begin{array}{l}n \\
n\end{array}$ & 串总 & - & 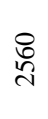 & 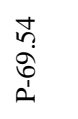 & $\begin{array}{l}8 \\
8\end{array}$ & 1 \\
\hline$\sqrt[\widetilde{\Omega}]{\approx}$ & 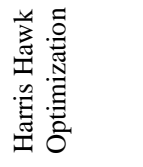 & - & 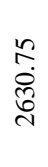 & 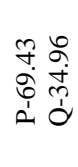 & $\frac{ \pm}{8}$ & $\begin{array}{l}\hat{\alpha} \\
\hat{\sigma}\end{array}$ \\
\hline $\begin{array}{l}\bar{\sigma} \\
\stackrel{2}{n}\end{array}$ & 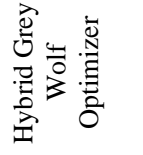 & $=$ & $\underset{\infty}{\infty}$ & $\begin{array}{l}\stackrel{n}{\Xi} \\
\stackrel{n}{+} \\
\frac{1}{2}\end{array}$ & $\begin{array}{l}\stackrel{n}{n} \\
m\end{array}$ & $\frac{\bar{\sigma}}{\sigma}$ \\
\hline$\stackrel{\widetilde{\sim}}{\approx}$ & 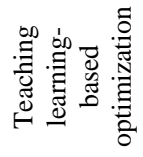 & $=$ & $\begin{array}{l}\text { ले } \\
\stackrel{0}{\infty} \\
\infty\end{array}$ & 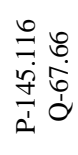 & $\begin{array}{l}n \\
m\end{array}$ & $\frac{\Delta}{\sigma}$ \\
\hline$\sqrt{n}$ & 包芯芯 & $\exists$ & $\begin{array}{l}\mathscr{8} \\
8 \\
\stackrel{1}{1} \\
m\end{array}$ & $\begin{array}{l}\bar{b} \\
\dot{+} \\
\dot{1}\end{array}$ & $\frac{n}{\hat{\sigma}}$ & I \\
\hline$\sqrt[\widetilde{\Omega}]{\underline{n}}$ & 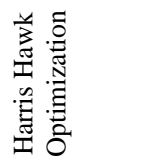 & 目 & $\begin{array}{l}m \\
m \\
\infty \\
\infty \\
\infty\end{array}$ & $\begin{array}{ll}\text { ஸे } \\
\stackrel{+}{1} \\
\stackrel{1}{1} \\
\stackrel{d}{0}\end{array}$ & $=$ & $\begin{array}{l}\tilde{a} \\
\stackrel{\sigma}{\sigma}\end{array}$ \\
\hline
\end{tabular}

The study shows that optimal placement of DG not only helps in reduction of power losses but also enhance the system voltage along with reduction in other factors such as voltage sag, total harmonic distortion etc. which can be harmful for system reliability and operation.

The ideal location for DG placement is observed by

$>$ Selection of objective(s)

$>$ Identifying system constraints

$>$ Selection of optimization method

$>$ Identifying the type of load

$>$ Accordingly, identifying the type of DG required.

$>$ Obtaining results and comparing

Various methods have been studied to identify the best location for placing DG in order to obtain maximum benefit from it. DG's scattered all over the system are more efficient as compared to those concentrated at one location.

\section{CONCLUSION}

This paper presents a brief review on optimal placement of DG for minimizing power losses in the distribution system. It is observed that there are various factors on the basis of which the distributed generators can be placed in the system. Also, there are different types of optimization techniques which are used to analyse the system performance and hence helps in finding an ideal location to site DG. Further, a comparative study shows the results of power loss minimization analysis performed on 33 and 69 bus test system. It can be concluded that DG improves the system performance when placed optimally and integrated up to a certain penetration limit. It helps in enhancing system voltage profile, reduce power losses, increase system reliability and reduce cost when sized properly

\section{REFERENCES}

[1] P. Dinakara Prasad Reddy, V.C. Veera Reddy, T. Gowri Manohar, "Optimal renewable resources placement in distribution networks bycombined power loss index and whale optimization algorithms", Journal of Electrical Systems and Information Technology 5, 2018

[2] K. Balamurugan, Dipti Srinivasan, Thomas Reindl, "Impact of Distributed Generation on Power Distribution Systems", PV Asia Pacific Conference, 2011

[3] Donal Caples, SretoBoljevic, Michael F. Conlon, "Impact of Distributed Generation on Voltage Profile in $38 \mathrm{kV}$ Distribution System", International Conference on the European Energy Market (EEM), 2011

[4] M.Saber, F.M.A.Ghali, E.E.AbouElzahab, "The Effect Of DG Penetration On Short Circuit Currents Level" ,Intl Conf on Advanced Control Circuits Systems (ACCS) Systems \& 2017 Intl Conf on New Paradigms in Electronics \& Information Technology (PEIT), 2017

[5] Neenu Rose Antony, Sarin Baby, "Optimal DG Placement Considering Voltage Stability Enhancement Using PSO”, International Conference on Control Communication and Computing, 2013

[6] Satish Kumar Injeti, Vinod Kumar Thunuguntla, "Optimal integration of DGs into radial distribution network in the presence of plug-in electric vehicles to minimize daily active power losses and to improve the voltage profile of the system using bioinspired optimization algorithms", Protection and Control of Modern Power Systems, 2020

[7] Mir Emad Hamidi, Reza Mohammadi Chabanloo, "Optimal Allocation of Distributed Generation With Optimal Sizing of Fault Current Limiter to Reduce the Impact on Distribution Networks Using NSGA-II", IEEE SYSTEMS JOURNAL, 2019

[8] M.M. Aman, G.B. Jasmon, H. Mokhlis, A.H.A. Bakar, "Optimal placement and sizing of a DG based on a new power stability index and line losses", Electrical Power and Energy Systems, 2012

[9] GaliveetiHemakumar Reddy, Pranju Chakrapani, Arup Kumar Goswami, Nalin B. Dev Choudhury, "Optimal distributed generation placement in distribution system to improve reliability and critical loads pick up after natural disasters", Engineering Science and Technology, an International Journal, 2017

[10] Pankita Mehta, Praghnesh Bhatt, Vivek Pandya, "Optimal selection of distributed generating units and its placement for voltage stability enhancement and energy loss minimization", Ain Shams Engineering Journal, 2018

[11] Abdurrahman ShuaibuHassan, Yanxia Sun, Zenghui Wang, "Multi-objective for optimal placement and sizing DG units in reducingloss of power and enhancing voltage profile using BPSO-SLFA", Energy Reports, 2020

[12] AfrozAlam, Bushra Zaheer, Mohammad Zaid, "Optimal Placement of DG in Distribution System for Power Loss Minimization and Voltage Profile Improvement", International Conference on Computing, Power and Communication Technologies, 2018

[13] Waseem Haider, S Jarjees Ul Hassan, ArifMehdi ,Arif Hussain, Gerardo Ondo Micha Adjayeng, Chul-Hwan Kim, "Voltage Profile Enhancement and Loss Minimization Using 
Optimal Placement and Sizing of Distributed Generation in Reconfigured Network", Machines, 2021

[14] Su Hlaing Win, Pyone Lai Swe, "Loss Minimization of Power Distribution Network using Different Types of Distributed Generation Unit", International Journal of Electrical and Computer Engineering, 2015

[15] Oscar Andrew Zongo, Anant Oonsivilai, "Optimal placement of distributed generator for power loss minimization and voltage stability improvement", International Conference on Alternative Energy in Developing Countries and Emerging Economies, 2017

[16] Duan Jiandong, Yang Xuying, Yang Wenyu, "Study on Power Flow Calculation and Voltage Profile in Distribution System with Distributed Generation", Asia-Pacific Power and Energy Engineering Conference, 2009

[17] DavoodKhani, Ahmad Sadeghi Yazdankhah, Hossein Madadi Koja, "Impacts of distributed generations on power system transient and voltage stability", Electrical Power and Energy Systems, 2012

[18] Srinivasa Rao Gampa, D. Das, "Optimum placement and sizing of DGs considering average hourly variations of load", Electrical Power and Energy Systems, 2015

[19] Jiang Le, YangQiang, "Maximizing DG Penetration in Power Distribution Networks Based on Cost/Benefit Analysis", 35th Chinese Control Conference, 2016

[20] Purva Shanna, Ankush Tandon, "Techniques for Optimal Placement of DG in Radial Distribution System: A Review", International Conference on Communication, Control and Intelligent Systems, 2015

[21] Juma S. A, Muriithi C. M, Ngoo L. M, "Optimal Switching Sequence using a Metaheuristic Algorithm for Feeder Reconfiguration", International Journal of Engineering Research and Technology, 2018

[22] Tuba Gözel, M. HakanHocaoglu, "An analytical method for the sizing and siting of distributed generators in radial systems", Electric Power Systems Research, 2009

[23] Ambika Ramamoorthy, Rajeswari Ramachandran, "Optimal Siting and Sizing of Multiple DG Units for the Enhancement of Voltage Profile and Loss Minimization in Transmission Systems Using Nature Inspired Algorithms", The Scientific World Journal, 2016

[24] M. Sedighizadeh, M. Fallahnejad, M. R. Alemi, M. Omidvaran, D. Arzaghi-haris, "Optimal Placement of Distributed Generation Using Combination of PSO and Clonal Algorithm", International Conference on Power and Energy, 2010

[25] Chandrasekaran Venkatesan, Raju Kannadasan, Mohammed H. Alsharif, Mun-Kyeom Kim, Jamel Nebhen, "A Novel Multiobjective Hybrid Technique for Siting and Sizing of Distributed Generation and Capacitor Banks in Radial Distribution Systems", Sustainability, 2021

[26] M. Afzalan; M. A. Taghikhani; M. Sedighizadeh, "Optimal DG placement and sizing with PSO\&HBMO algorithm in radial distribution networks", 2012 Proceedings of 17th Conference on Electrical Power Distribution, 2012

[27] M C V Suresh, J. Belwin Edward, "A Hybrid Algorithm Based Optimal Placement of DG Units for Loss Reduction in the Distribution System", Applied Soft Computing Journal, 2020

[28] Jordan Radosavljević, NebojšaArsić, MilošMilovanović, Aphrodite Ktena, "Optimal Placement and Sizing of Renewable Distributed Generation Using Hybrid Metaheuristic Algorithm", Journal Of Modern Power Systems And Clean Energy, 2020

[29] Mohamed A. Tolba, Vladimir N. Tulsky, Ahmed A. Zaki Diab, "Optimal Sitting and Sizing of Renewable Distributed Generations in Distribution Networks Using a Hybrid PSOGSA Optimization Algorithm", IEEE International Conference on Environment and Electrical Engineering and 2017 IEEE Industrial and Commercial Power Systems Europe (EEEIC / I\&CPS Europe), 2017
[30] Sandeep Kaur, G. B. Kumbhar, Jaydev Sharma, "Harmony Search and OPF Based Hybrid Approach for Optimal Placement of Multiple DG Units", Eighteenth National Power Systems Conference (NPSC), 2014

[31] Mohammed Kdair Abd, S. J. Cheng, H. S. Sun, "Optimal DG Placement and Sizing for Power Loss Reduction in a Radial Distribution System using MPGSA and Sensitivity Index Method", 11th Conference on Industrial Electronics and Applications (ICIEA), 2016

[32] Duong Quoc Hung, N. Mithulananthan, Kwang Y. Lee, "Optimal placement of dispatchable and nondispatchable renewable DG units in distribution networks for minimizing energy loss", Electrical Power and Energy Systems, 2014

[33] A.N. Swathi, G. Venkata Suresh Babu, "Optimal Placement of Distributed Generation (Dg) Sources in Power Systems for Loss Reduction Using BIBC and Analytical Approach", International Journal of Research Studies in Science, Engineering and Technology, 2014

[34] M. Reza, P. H. Schavemaker, J. G. Slootweg, W. L. Kling, L. van der Sluis, "Impacts of Distributed Generation Penetration Levels on Power Systems Transient Stability", IEEE Power Engineering Society General Meeting, 2004

[35] KrischonmeBhumkittipich, WeerachaiPhuangpornpitak, "Optimal Placement and Sizing of Distributed Generation for Power Loss Reduction using Particle Swarm Optimization", 10th Eco-Energy and Materials Science and Engineering, 2012

[36] D.B. Prakash, C. Lakshminarayana, "Multiple DG Placements in Distribution System for Power Loss Reduction Using PSO Algorithm", Global Colloquium in Recent Advancement and Effectual Researches in Engineering, Science and Technology, 2016

[37] Ni KetutAryani, Muhammad Abdillah, I Made Yulistya Negara, Adi Soeprijanto, "Optimal Placement and Sizing of Distributed Generation using Quantum Genetic Algorithm for Reducing Losses and Improving Voltage Profile", IEEE Region 10 Conference, 2011

[38] Mohan Kashyap, Ankit Mittal, Satish Kansal, "Optimal Placement of Distributed Generation Using Genetic Algorithm Approach", Proceeding of the Second International Conference on Microelectronics, Computing \& Communication systems, 2019

[39] Rashmi Deshmukh, Amol Kalage, "Optimal Placement and Sizing of Distributed Generator in Distribution System Using Artificial Bee Colony Algorithm", IEEE Global Conference on Wireless Computing and Networking, 2018

[40] Snigdha Rani Behera, Soumya Prakash Dash, B. K. Panigrahi, "Optimal Placement and Sizing of DGs in Radial Distribution System (RDS) using Bat Algorithm", International Conference on Circuit, Power and Computing Technologies, 2015

[41] Mark NdubukaNwohu, LanreOlatomiwa, James Ambafi, Sadiq Abubakar Ahmad, Abdullahi Mogaji, "Optimal Deployment of Distributed Generators using Ant Colony Optimization to Minimize Line Losses and Improve Voltage Profiles on Distribution Network", Proceedings of the World Congress on Engineering and Computer Science, 2017

[42] Chandrasekhar Yammani, SyduluMaheswarapu, SailajakumariMatam, "Multiobjective Optimization for Optimal Placement and Size of DG using Shuffled Frog Leaping Algorithm", 2nd International Conference on Advances in Energy Engineering, 2012

[43] Ram Prakash, B.C. Sujatha, "Optimal Placement and Sizing of DG for Power Loss Minimization and VSI Improvement using Bat Algorithm", 19th National Power Systems Conference, 2016

[44] Sara YuliethBocanegra, Oscar Danilo Montoya, "Heuristic Approach for Optimal Location and Sizing of Distributed Generators in AC Distribution Networks", WSEAS Transactions on Power systems, 2019

[45] SheerazKirmani, Majid Jamil, M. Rizwan, "Optimal Placement of SPV based DG System for Loss Reduction in Radial Distribution Network Using Heuristic Search 
Strategies", International Conference on Energy, Automation and Signal, 2011

[46] Caisheng Wang, M. Hashem Nehrir, "Analytical Approaches for Optimal Placement of Distributed Generation Sources in Power Systems", IEEE Transactions On Power Systems,2004

[47] ParthaKayal, Sayonsom Chanda, Chandan Kumar Chanda, "An analytical approach for allocation and sizing of distributed generations in radial distribution network", Int Trans ElectrEnerg Syst., 2017

[48] ParthaKayal, C.K. Chanda, "Placement of wind and solar based DGs in distribution system for power loss minimization and voltage stability improvement", Electrical Power and Energy Systems, 2013

[49] P. Vijay Babu, S.P. Singh, "Optimal Placement of DG in Distribution network for Power loss minimization using NLP \& PLS Technique", 5th International Conference on Advances in Energy Research, 2016

[50] S. Gopiya Naik, D.K. Khatod, M.P. Sharma, "Optimal allocation of combined DG and capacitor for real power loss minimization in distribution networks", Electrical Power and Energy Systems, 2013

[51] YuvarajThangaraj, Ravi Kuppan, "Multi-objective simultaneous placement of DG and DSTATCOM usingnovel lightning search algorithm", Journal of Applied Research and Technology, 2017

[52] Ponnam Venkata K. Babu, K. Swarnasri, "Optimal integration of different types of DGs inradial distribution system by using Harris hawkoptimization algorithm", Cogent Engineering, 2020

[53] V. V. V. S. N.Murty,A. Kumar, "Optimal DGintegration and network reconfiguration in microgridsystem with realistic time varying load model usinghybrid optimisation", IET Smart Grid, 2019

[54] M. C. V.Suresh,E. J.Belwin, "Optimal DG placementfor benefit maximization in distribution networksby using
Dragonfly algorithm", Renewables:Wind, Water and Solar, 2018

[55] Kansal S., Kumar V., B. Tyagi, “Hybrid approachfor optimal placement of multiple DGs of multipletypes in distribution networks", International Journalof Electrical Power \& Energy Systems, 2016

[56] R. Sanjay, T. Jayabarathi, T. Raghunathan, V. Ramesh, N. Mithulananthan, "Optimal allocation ofdistributed generation using hybrid Grey Wolf optimizer", IEEE Access, 2017

[57] K. Muthukumar, S. Jayalalitha, "Optimal placementand sizing of distributed generators and shunt capacitorsfor power loss minimization in radial distribution networksusing hybrid heuristic search optimization technique", International Journal of Electrical Power \& Energy Systems, 2016

[58] S. Kaur, G. KumbharJ. Sharma, "A MINLPtechnique for optimal placement of multiple DG unitsin distribution systems", International Journal ofElectrical Power \& Energy Systems, 2014

[59] S.A. ChithraDevi, L. Lakshminarasimman, R. Balamurugan "Study Krill herd algorithm for multiple DGplacement and sizing in a radial distribution system", Engineering Science and Technology, 2017

[60] D. B. Prakash, C.Lakshminarayana, "Multiple DGplacements in radial distribution system for multiobjectives using whale optimization algorithm", Alexandria Engineering Journal, 2018

[61] V. Tamilselvan, T. Jayabarathi, T. Raghunathan, X. S. Yang, "Optimal capacitor placement inradial distribution systems using flower pollinationalgorithm", Alexandria Engineering Journal, 2018

[62] C. H. Prasad, K. Subbaramaiah, P. Sujatha, "Cost-benefit analysis for optimal DG placement in distributionsystems by using elephant herding optimization algorithm", Renewables: Wind, Water and Solar, 2019 